(C) [2006] IEEE. Reprinted, with permission, from [Jian Xun Jin,You Guang Guo and Jian Guo Zhu Chang Ming Zhang, Power Electronic-Controlled High Q Resonator Theory with HTS Technology, Communications, Circuits and Systems Proceedings, 2006 International Conference on (Volume:4 ) 25-28 June 2006]. This material is posted here with permission of the IEEE. Such permission of the IEEE does not in any way imply IEEE endorsement of any of the University of Technology, Sydney's products or services. Internal or personal use of this material is permitted.

However, permission to reprint/republish this material for advertising or promotional purposes or for creating new collective works for resale or redistribution must be obtained from the IEEE by writing to pubs-permissions@ieee.org. By choosing to view this document, you agree to all provisions of the copyright laws protecting it 


\section{Power Electronic-Controlled High Q Resonator Theory with HTS Technology}

\author{
Jian Xun Jin $^{1}$, and Chang Ming Zhang ${ }^{2}$ \\ ${ }^{1}$ Center of Applied Superconductivity and Electrical Eng., \\ School of Automation Engineering; \\ ${ }^{2}$ School of Optoelectronic Information, \\ University of Electronic Science \& Technology of China, \\ Chengdu, China 610054
}

\author{
You Guang Guo, and Jian Guo Zhu \\ School of Electrical Engineering, \\ Faculty of Engineering, \\ University of Technology Sydney, \\ Broadway, Sydney, \\ NSW 2007, Australia
}

\begin{abstract}
Power electronic-controlled switch has been used with a high-temperature superconductor (HTS) to develop an advanced high $Q$ resonant circuit. With a HTS, a very high $Q$ circuit can be achieved; consequently special aspects such as high voltage generation can be theoretically and practically realized. Theoretical study has been carried out for this concept and method, and this paper describes the theory of this high $Q$ resonant circuit and the operational principle.
\end{abstract}

\section{INTRODUCTION}

An electronic resonant circuit with a power electroniccontrolled switch has been explored with regard to its special aspects of using a high $\mathrm{T}_{\mathrm{c}}$ superconductor (HTS) [1,2]. The circuit special aspects have been studied, and especially to form a method of very high voltage generation from a low voltage source. This high voltage generation method using the high Q resonant circuit mainly consists of a HTS inductor, a DC battery source, a high voltage capacitor, and an electronic switch. As a basic principle of operational approach, a low voltage DC power source can be used and its polarity is reversed at a certain frequency and this control is achieved with an electronic switch. As a fundamental principle, the resonant circuit generates the voltage which is proportional to the circuit $\mathrm{Q}$ value. Resistance in the circuit will limit the $\mathrm{Q}$ value and therefore voltages that can be achieved in practice; however HTS technology can dramatically reduce the resistance and present a very high $\mathrm{Q}$ value in its circuit.

With regard to the power electronic-controlled HTS high $\mathrm{Q}$ resonator, both theoretical analysis and practical operation principle will be presented in this paper with the advantages of potentially using a high $\mathrm{Q}$ electronic circuit which can be made with HTS Ag-clad $(\mathrm{Bi}, \mathrm{Pb})_{2} \mathrm{Sr}_{2} \mathrm{Ca}_{2} \mathrm{Cu}_{3} \mathrm{O}_{10+x}$ multifilament wires.

\section{CIRCUIT PRINCIPLE}

\section{A. Q Value and Voltage Feature of a Resonant Circuit}

In a resistive RCL series resonant circuit, the quality factor of a resonant circuit is $\mathrm{Q}=\omega_{0} \mathrm{~L} / \mathrm{R}(=\mathrm{X} / \mathrm{R})$, and $\omega_{0}=$ $\left[(1 / \mathrm{LC})-\left(\mathrm{R}^{2} / 4 \mathrm{~L}^{2}\right)\right]^{1 / 2}$ is the resonant frequency in $\mathrm{rad} \mathrm{s}^{-1}$. The potential difference across the capacitor $\mathrm{V}_{\mathrm{c}}$ at resonance is $\mathrm{Q}$ times as great as the applied emf $V_{p}(r m s)$ to the circuit. For a sinusoidal power supply, the voltage across the capacitor $\mathrm{V}_{\text {Cmax }}$ at resonance is given by: $\mathrm{V}_{\mathrm{Cmax}}=\mathrm{Q} \mathrm{V}_{\mathrm{p}}$. For using a DC power supply with an electronic switch to reverse the polarity, the maximum voltage $\mathrm{V}_{\mathrm{Cmax}}$ can be expressed as: $\mathrm{V}_{\text {Cmax }}=\mathrm{Q} \mathrm{V}_{\mathrm{B}}$, where $\mathrm{V}_{\mathrm{B}}$ ' is the effective voltage of the low voltage power source. If the switching controller used has rectangular switching wave form with low voltage source polarity switching frequency $f$, the build-up voltage wave form $F(t)$ can be expressed by Fourier series as

$$
F(t)=\left(4 V_{B} / \pi\right)[\sin \omega t+(1 / 3) \sin 3 \omega t+(1 / 5) \sin 5 \omega t+\ldots]
$$

The generator only resonates on the first harmonic with amplitude of $4 \mathrm{~V}_{\mathrm{B}} / \pi$. Therefore, $\mathrm{V}_{\mathrm{B}}{ }^{\prime}=4 \mathrm{~V}_{\mathrm{B}} / \pi$, and the maximum build-up voltage is related with the RCL resonant circuit $\mathrm{Q}$ value by: $\mathrm{V}_{\mathrm{Cmax}}=\mathrm{Q}\left(4 \mathrm{~V}_{\mathrm{B}} / \pi\right)$. Reducing the RCL resonant circuit resistance is achieved by introducing the superconducting inductor; the circuit $\mathrm{Q}$ value then will be dramatically increased, therefore leading to a very high voltage across the capacitor.

\section{B. Resistance-Less Circuit}

For the RCL resonant circuit, when a DC source is switched to the circuit, the instantaneous current $\mathrm{i}(\mathrm{t})$ and capacitor voltage $\mathrm{V}_{\mathrm{C}}(\mathrm{t})$ solutions respectively are 


$$
\begin{array}{r}
\mathrm{i}(\mathrm{t})=\mathrm{e}^{\frac{-\mathrm{Rt}}{2 \mathrm{~L}}}\left[\frac{\left(\mathrm{V}_{\mathrm{CO}}+\mathrm{V}_{\mathrm{B}}\right) \sin \omega \mathrm{t}}{\omega \mathrm{L}}\right] \\
\mathrm{V}_{\mathrm{C}}(\mathrm{t})=\left(\mathrm{V}_{\mathrm{CO}}+\mathrm{V}_{\mathrm{B}}\right)\left[1-\mathrm{e}^{\frac{-\mathrm{Rt}}{2 \mathrm{~L}}}\left(\cos \omega \mathrm{t}+\frac{\mathrm{R}}{2 \mathrm{~L} \omega} \sin \omega \mathrm{t}\right)\right]-\mathrm{V}_{\mathrm{CO}}
\end{array}
$$

where $\mathrm{R}<2(\mathrm{~L} / \mathrm{C})^{1 / 2}, \mathrm{~V}_{\mathrm{CO}}$ is the initial capacitor voltage. Both equations describe decaying sinusoids, with $V_{C}(t)$ approaching a steady state value of $\mathrm{V}_{\mathrm{B}}$, and $\mathrm{i}(\mathrm{t})$ approaching a steady state value of zero.

Now in a circuit using a superconducting inductor and no separate resistor, then $\mathrm{R}$ will become very small. If $\mathrm{R}=0 \Omega$, then (2) and (3) can be simplified to

$$
\begin{gathered}
\mathrm{i}(\mathrm{t})=\frac{\left(\mathrm{V}_{\mathrm{CO}}+\mathrm{V}_{\mathrm{B}}\right) \sin \omega \mathrm{t}}{\omega \mathrm{L}} \\
\mathrm{V}_{\mathrm{C}}(\mathrm{t})=-\left(\mathrm{V}_{\mathrm{CO}}+\mathrm{V}_{\mathrm{B}}\right) \cos \omega \mathrm{t}+\mathrm{V}_{\mathrm{B}}
\end{gathered}
$$

where $\omega=(\mathrm{LC})^{-1 / 2} \mathrm{rad} \mathrm{s}^{-1}$. These two equations describe constant magnitude sinusoids, with the average values of $\mathrm{i}(\mathrm{t})$ and $\mathrm{V}_{\mathrm{C}}(\mathrm{t})$ being zero and $\mathrm{V}_{\mathrm{B}}$ respectively.

When the DC source is switched in, $V_{C}(t)=V_{C}(0)=-$ $\mathrm{V}_{\mathrm{CO}}$. One half a resonant cycle later, this voltage will have increased to

$$
\mathrm{V}_{\mathrm{C}}(\mathrm{t})=\mathrm{V}_{\mathrm{C}}(\pi / \omega)=-\left(\mathrm{V}_{\mathrm{CO}}+\mathrm{V}_{\mathrm{B}}\right)(-1)+\mathrm{V}_{\mathrm{B}}=\mathrm{V}_{\mathrm{CO}}+2 \mathrm{~V}_{\mathrm{B}}
$$

If at this point of time the DC source is disconnected, and then reconnected in the opposite polarity for the next half cycle, then the initial capacitor voltage $V_{\mathrm{CO}}$ is changed to $\mathrm{V}_{\text {CO-new, }}$ and is given by

$$
\mathrm{V}_{\mathrm{CO}-\mathrm{new}}=-\mathrm{V}_{\mathrm{C}}(\mathrm{t})=-\left(\mathrm{V}_{\mathrm{CO}}+2 \mathrm{~V}_{\mathrm{B}}\right)
$$

Half a cycle later, $\mathrm{V}_{\mathrm{C}}(\mathrm{t})=\mathrm{V}_{\mathrm{C}}(2 \pi / \omega)$ becomes

$$
\mathrm{V}_{\mathrm{C}}(\mathrm{t})=-\left[-\left(\mathrm{V}_{\mathrm{CO}}+2 \mathrm{~V}_{\mathrm{B}}\right)+\left(-\mathrm{V}_{\mathrm{B}}\right)\right](-1)+\left(-\mathrm{V}_{\mathrm{B}}\right)=-\left(\mathrm{V}_{\mathrm{CO}}+4 \mathrm{~V}_{\mathrm{B}}\right)
$$

If the DC source polarity is reversed every half cycle thereafter then

$$
\mathrm{V}_{\mathrm{C}}(\mathrm{t})=\left(\mathrm{V}_{\mathrm{CO}}+6 \mathrm{~V}_{\mathrm{B}}\right) ;-\left(\mathrm{V}_{\mathrm{CO}}+8 \mathrm{~V}_{\mathrm{B}}\right) ;\left(\mathrm{V}_{\mathrm{CO}}+10 \mathrm{~V}_{\mathrm{B}}\right) ; \ldots \text { etc }
$$

This is the build-up voltage for an ideal non-resistive circuit. Therefore the positive and negative peak voltages can be described by (9). Consequently in a resistance-less circuit, the voltage across the capacitor $\mathrm{C}$ after $\mathrm{n}$ cycles will be:

$$
V_{C}(n)=(-1)^{n+1}\left(V_{C O}+2 n V_{B}\right)
$$

where $\mathrm{n}$ is the iteration number, and $\mathrm{V}_{\mathrm{CO}}$ is the initial capacitor voltage.

\section{Practical Resistive Circuit}

From (3), when $t=\pi / \omega, i=0$, if the DC source changes polarity, the capacitor voltage is given by

$$
\mathrm{V}_{\mathrm{C} 1}=\left(\mathrm{V}_{\mathrm{CO}}+\mathrm{V}_{\mathrm{B}}\right)\left(1+\mathrm{e}^{-\mathrm{R}_{\pi} / 2 \mathrm{~L}_{\omega}}\right)-\mathrm{V}_{\mathrm{CO}}
$$

After the polarity is changed $\mathrm{n}$ times, the capacitor voltage becomes

$$
\mathrm{V}_{\mathrm{Cn}}=\left(\mathrm{V}_{\mathrm{Cn}-1}+\mathrm{V}_{\mathrm{B}}\right)\left(1+\mathrm{e}^{-\mathrm{R}_{\pi} / 2 \mathrm{~L}_{\omega}}\right)-\mathrm{V}_{\mathrm{Cn}-1}
$$

If $\mathrm{V}_{\mathrm{CO}}=0$, then

$$
\begin{aligned}
& \mathrm{V}_{\mathrm{C} 1}=\mathrm{V}_{\mathrm{B}}\left(1+\mathrm{e}^{-\mathrm{R}_{\pi} / 2 \mathrm{~L}_{\omega}}\right) \\
& \mathrm{V}_{\mathrm{C} 2}=\left(\mathrm{V}_{\mathrm{C} 1}+\mathrm{V}_{\mathrm{B}}\right)\left(1+\mathrm{e}^{-\mathrm{R}_{\pi} / 2 \mathrm{~L}_{\omega}}\right)-\mathrm{V}_{\mathrm{C} 1} \\
&=\mathrm{V}_{\mathrm{B}}\left(1+2 \mathrm{e}^{-\mathrm{R}_{\pi} / 2 \mathrm{~L}_{\omega}}+\mathrm{e}^{-2 \mathrm{R}_{\pi} / 2 \mathrm{~L}_{\omega}}\right) \\
& \ldots \ldots \\
& \mathrm{V}_{\mathrm{Cn}}=\left(\mathrm{V}_{\mathrm{Cn}-1}+\mathrm{V}_{\mathrm{B}}\right)\left(1+\mathrm{e}^{-\mathrm{R}_{\pi} / 2 \mathrm{~L}_{\omega}}\right)-\mathrm{V}_{\mathrm{Cn}-1} \\
&=\left(1+\mathrm{V}_{\mathrm{B}}\left(1+2 \mathrm{e}^{-\mathrm{R}_{\pi} / 2 \mathrm{~L}_{\omega}}+2 \mathrm{e}^{-2 \mathrm{R} / 2 \mathrm{~L}_{\omega}} \ldots+2 \mathrm{e}^{-(\mathrm{n}-1) \mathrm{R}_{\pi} / 2 \mathrm{~L}_{\omega}}+\mathrm{e}^{-\mathrm{nR}_{\pi} / 2 \mathrm{~L}_{\omega}}\right)+2 \mathrm{~V}_{\mathrm{B}} \sum_{\mathrm{i}=1}^{\mathrm{n}-1} \mathrm{e}^{-\mathrm{iR} \pi / 2 \mathrm{~L} \omega}\right.
\end{aligned}
$$

Assuming that the power supply polarity changes at $\mathrm{t}=$ $\mathrm{n} \pi / \omega$, after the polarity is changed $n$ times, the build-up $V_{C n}$ is given by

$$
\mathrm{V}_{\mathrm{Cn}}=\mathrm{V}_{\mathrm{B}}\left(1+\mathrm{e}^{-\mathrm{nR} \pi / 2 \mathrm{~L} \omega}\right)+2 \mathrm{~V}_{\mathrm{B}} \frac{\mathrm{e}^{-\mathrm{R} \pi / 2 \mathrm{~L} \omega}-\mathrm{e}^{-(\mathrm{n}-1) \mathrm{R} \pi / 2 \mathrm{~L} \omega}}{1-\mathrm{e}^{-\mathrm{R} \pi / 2 \mathrm{~L} \omega}}
$$

When $\mathrm{n} \rightarrow \propto, \mathrm{V}_{\mathrm{Cn}} \rightarrow \mathrm{V}_{\mathrm{Cmax}}$, therefore

$$
\mathrm{V}_{\mathrm{Cmax}}=\lim _{\mathrm{n} \rightarrow \infty} \mathrm{V}_{\mathrm{Cn}}=\mathrm{V}_{\mathrm{B}}\left(1+\frac{2}{\mathrm{e}^{\mathrm{R} \pi / 2 \mathrm{~L} \omega}-1}\right)
$$

From (16), when $\mathrm{R} \rightarrow 0, \mathrm{~V}_{\mathrm{Cmax}} \rightarrow \propto$. When $\mathrm{R} \rightarrow \propto, \mathrm{V}_{\mathrm{Cmax}}$ $\rightarrow V_{B}$. Since $Q=\omega L / R$, therefore (16) can be expressed as

$$
\mathrm{V}_{\text {Cmax }}=\mathrm{VB}_{\mathrm{B}}+\frac{2 \mathrm{VB}_{\mathrm{B}}}{\mathrm{e}^{\pi / 2 \mathrm{Q}}-1}
$$

\section{Step Voltage Source}

The low voltage sources can be an ac power supply, a dc battery or a rectangular pulse source. When a rectangular pulse source $\mathrm{u}_{\mathrm{s}}$ is used, consequently the RCL circuit has its differential equation $\mathrm{LC} \frac{\mathrm{d}^{2} \mathrm{v}_{\mathrm{c}}}{\mathrm{dt}^{2}}+\mathrm{RC} \frac{\mathrm{dv_{ \textrm {c } }}}{\mathrm{dt}}+\mathrm{v}_{\mathrm{c}}=\mathrm{u}_{\mathrm{s}}$ where its characteristic roots are $S_{1,2}=-\frac{R}{2 L} \pm \sqrt{\left(\frac{R}{2 L}\right)^{2}-\frac{1}{L C}}$, 
if $\mathrm{R}^{2}<\frac{4 \mathrm{~L}}{\mathrm{C}}$, then its natural frequencies are conjugate complex numbers,

$S_{1,2}=-\frac{R}{2 L} \pm j \sqrt{\frac{1}{L C}-\left(\frac{R}{2 L}\right)^{2}}=-\alpha \pm j \omega_{d}$, where $\alpha=\frac{R}{2 L}$ is attenuation coefficient, $\omega_{\mathrm{d}}=\sqrt{\frac{1}{\mathrm{LC}}-\left(\frac{\mathrm{R}}{2 \mathrm{~L}}\right)^{2}}$ is damping angular frequency. $\omega_{\mathrm{d}}=\sqrt{\omega_{0}{ }^{2}-\alpha^{2}}$, where $\omega_{0}=\frac{1}{\sqrt{\mathrm{LC}}}$ is series resonant angular frequency.

If the exciting source is a step voltage source, $\mathrm{u}_{\mathrm{s}}=$ $\mathrm{V}_{\mathrm{s}} \varepsilon(\mathrm{t})$, then $\mathrm{u}_{\mathrm{s}}=\mathrm{v}_{\mathrm{c}}(\mathrm{t})=\mathrm{e}^{-\alpha \mathrm{t}}\left(\mathrm{K}_{1} \cos \omega_{\mathrm{d}} \mathrm{t}+\mathrm{K}_{2} \sin \omega_{\mathrm{d}} \mathrm{t}\right)+\mathrm{V}_{\mathrm{s}}$. If the circuit has zero initial state, then $\mathrm{v}_{\mathrm{c}}(0)=\mathrm{K}_{1}+\mathrm{V}_{\mathrm{s}}=0$, $\left.\frac{\mathrm{dv}_{\mathrm{c}}(\mathrm{t})}{\mathrm{dt}}\right|_{\mathrm{t}=0}=-\alpha \mathrm{K}_{1}+\omega_{\mathrm{d}} \mathrm{K}_{2}=0$; when $\mathrm{K}_{1}=-\mathrm{V}_{\mathrm{s}}, \mathrm{K}_{2}=-$ $\mathrm{V}_{\mathrm{s}} \alpha / \omega_{\mathrm{d}}$, then

$$
\begin{aligned}
& \mathrm{v}_{\mathrm{C}}(\mathrm{t})=-\mathrm{V}_{\mathrm{S}} \mathrm{e}^{-\alpha \mathrm{t}}\left(\cos \omega_{\mathrm{d}} \mathrm{t}+\frac{\alpha}{\omega_{\mathrm{d}}} \sin \omega_{\mathrm{d}} \mathrm{t}\right)+\mathrm{V}_{\mathrm{S}}=\mathrm{V}_{\mathrm{S}}\left[1-\mathrm{e}^{-\alpha \mathrm{t}} \sqrt{1+\frac{\alpha^{2}}{\omega_{\mathrm{d}}^{2}}}\left(\frac{1}{\sqrt{1+\frac{\alpha^{2}}{\omega_{\mathrm{d}}{ }^{2}}}} \cos \omega_{\mathrm{d}} \mathrm{t}+\frac{\alpha}{\omega_{\mathrm{d}} \sqrt{1+\frac{\alpha^{2}}{\omega_{\mathrm{d}}{ }^{2}}}} \sin \omega_{\mathrm{d}} \mathrm{t}\right]\right) \\
& =\mathrm{V}_{\mathrm{s}}\left[1-\frac{\omega_{0}}{\omega_{\mathrm{d}}} \mathrm{e}^{-\alpha \mathrm{t}} \cos \left(\omega_{\mathrm{d}} \mathrm{t}-\theta\right)\right], \quad \mathrm{t} \geq 0, \quad\left(\theta=\operatorname{arctg} \frac{\alpha}{\omega_{\mathrm{d}}}\right)
\end{aligned}
$$

If exciting source is a causality pulse voltage source

$$
\mathrm{u}_{\mathrm{s}}=\varepsilon(\mathrm{t})-2 \varepsilon\left(\mathrm{t}-\frac{\mathrm{T}}{2}\right)+2 \varepsilon(\mathrm{t}-\mathrm{T})-2 \varepsilon\left(\mathrm{t}-\mathrm{T}-\frac{\mathrm{T}}{2}\right)+2 \varepsilon(\mathrm{t}-2 \mathrm{~T})-2 \varepsilon\left(\mathrm{t}-2 \mathrm{~T}-\frac{\mathrm{T}}{2}\right)+2 \varepsilon(\mathrm{t}-3 \mathrm{~T}) \ldots \ldots
$$

for the first cycle, $\mathrm{u}_{\mathrm{s}}(\mathrm{t})=\left\{\begin{array}{l}\varepsilon(\mathrm{t}), \quad 0<\mathrm{t}<\frac{\mathrm{T}}{2} \\ \varepsilon(\mathrm{t})-2 \varepsilon\left(\mathrm{t}-\frac{\mathrm{T}}{2}\right), \quad \frac{\mathrm{T}}{2}<\mathrm{t}<\mathrm{T}\end{array}\right.$

after the first cycle,

$$
\mathrm{u}_{\mathrm{s}}(\mathrm{t})=\left\{\begin{array}{l}
\varepsilon(\mathrm{t})-2 \sum_{\mathrm{k}=1}^{\mathrm{k}} \varepsilon\left[\mathrm{t}-(\mathrm{k}-1) \mathrm{T}-\frac{\mathrm{T}}{2}\right]+2 \sum_{\mathrm{k}=1}^{\mathrm{k}} \varepsilon(\mathrm{t}-\mathrm{kT}), \quad \mathrm{kT}<\mathrm{t}<\mathrm{kT}+\frac{\mathrm{T}}{2} \\
\varepsilon(\mathrm{t})-2 \sum_{\mathrm{k}=1}^{\mathrm{k}} \varepsilon\left[\mathrm{t}-(\mathrm{k}-1) \mathrm{T}-\frac{\mathrm{T}}{2}\right]+2 \sum_{\mathrm{k}=1}^{\mathrm{k}} \varepsilon(\mathrm{t}-\mathrm{kT})-2 \varepsilon\left(\mathrm{t}-\mathrm{kT}-\frac{\mathrm{T}}{2}\right), \quad \mathrm{kT}+\frac{\mathrm{T}}{2}<\mathrm{t}<(\mathrm{k}+1) \mathrm{T}
\end{array}\right.
$$

$\mathrm{V}_{\mathrm{C}}$ in the first cycle,

$$
\mathrm{V}_{\mathrm{c}}(\mathrm{t})=\left\{\begin{array}{l}
\mathrm{V}_{\mathrm{s}}\left[1-\frac{\omega_{0}}{\omega_{\mathrm{d}}} \mathrm{e}^{-\alpha \mathrm{t}} \cos \left(\omega_{\mathrm{d}} \mathrm{t}-\theta\right)\right], \quad 0<\mathrm{t}<\frac{\mathrm{T}}{2} ; \\
\mathrm{V}_{\mathrm{s}}\left[1-\frac{\omega_{0}}{\omega_{\mathrm{d}}} \mathrm{e}^{-\alpha \mathrm{t}} \cos \left(\omega_{\mathrm{d}} \mathrm{t}-\theta\right)\right]-2 \mathrm{~V}_{\mathrm{s}}\left[1-\frac{\omega_{0}}{\omega_{\mathrm{d}}} \mathrm{e}^{-\alpha\left(\mathrm{t}-\frac{\mathrm{T}}{2}\right)} \cos \left(\omega_{\mathrm{d}}\left(\mathrm{t}-\frac{\mathrm{T}}{2}\right)-\theta\right)\right], \quad \frac{\mathrm{T}}{2}<\mathrm{t}<\mathrm{T}
\end{array}\right.
$$


After the first cycle,

$$
\mathrm{v}_{\mathrm{c}}(\mathrm{t})=\left\{\begin{array}{l}
\mathrm{V}_{\mathrm{s}}\left[1-\frac{\omega_{0}}{\omega_{\mathrm{d}}} \mathrm{e}^{-\alpha \mathrm{t}} \cos \left(\omega_{\mathrm{d}} \mathrm{t}-\theta\right)\right]-2 \sum_{\mathrm{k}=1}^{\mathrm{k}} \mathrm{V}_{\mathrm{s}}\left[1-\frac{\omega_{0}}{\omega_{\mathrm{d}}} \mathrm{e}^{-\alpha\left[\mathrm{t}-(\mathrm{k}-1) \mathrm{T}-\frac{\mathrm{T}}{2}\right]} \cos \left(\omega_{\mathrm{d}}\left[\mathrm{t}-(\mathrm{k}-1) \mathrm{T}-\frac{\mathrm{T}}{2}\right]-\theta\right)\right] \\
+2 \sum_{\mathrm{k}=1}^{\mathrm{k}} \mathrm{V}_{\mathrm{s}}\left[1-\frac{\omega_{0}}{\omega_{\mathrm{d}}} \mathrm{e}^{-\alpha(\mathrm{t}-\mathrm{kT})} \cos \left(\omega_{\mathrm{d}}(\mathrm{t}-\mathrm{kT})-\theta\right)\right], \quad \mathrm{kT}<\mathrm{t}<\mathrm{kT}+\frac{\mathrm{T}}{2} ; \\
\mathrm{V}_{\mathrm{s}}\left[1-\frac{\omega_{0}}{\omega_{\mathrm{d}}} \mathrm{e}^{-\alpha \mathrm{t}} \cos \left(\omega_{\mathrm{d}} \mathrm{t}-\theta\right)\right]-2 \sum_{\mathrm{k}=1}^{\mathrm{k}} \mathrm{V}_{\mathrm{s}}\left[1-\frac{\omega_{0}}{\omega_{\mathrm{d}}} \mathrm{e}^{-\alpha\left[\mathrm{t}-(\mathrm{k}-1) \mathrm{T}-\frac{\mathrm{T}}{2} \mathrm{~T}\right.} \cos \left(\omega_{\mathrm{d}}\left[\mathrm{t}-(\mathrm{k}-1) \mathrm{T}-\frac{\mathrm{T}}{2}\right]-\theta\right)\right] \\
+2 \sum_{\mathrm{k}=1}^{\mathrm{k}} \mathrm{V}_{\mathrm{s}}\left[1-\frac{\omega_{0}}{\omega_{\mathrm{d}}} \mathrm{e}^{-\alpha(\mathrm{t}-\mathrm{kT})} \cos \left(\omega_{\mathrm{d}}(\mathrm{t}-\mathrm{kT})-\theta\right)\right] \\
-2 \mathrm{~V}_{\mathrm{s}}\left[1-\frac{\omega_{0}}{\omega_{\mathrm{d}}} \mathrm{e}^{-\alpha\left(\mathrm{t}-\mathrm{kT}-\frac{\mathrm{T}}{2}\right)} \cos \left(\omega_{\mathrm{d}}\left(\mathrm{t}-\mathrm{kT}-\frac{\mathrm{T}}{2}\right)-\theta\right)\right], \quad \mathrm{kT}+\frac{\mathrm{T}}{2}<\mathrm{t}<(\mathrm{k}+1) \mathrm{T}
\end{array}\right.
$$

For the steady state after a number of cycles, (23) can be simplified as

$$
\mathrm{V}_{\mathrm{c}}(\mathrm{t}) \approx\left\{\begin{array}{l}
\mathrm{V}_{\mathrm{s}}-2 \mathrm{~V}_{\mathrm{s}} \frac{\omega_{0}}{\omega_{\mathrm{d}}} \mathrm{e}^{-\alpha(\mathrm{t}-\mathrm{kT})}\left[\mathrm{e}^{-\alpha \frac{\mathrm{T}}{2}} \cos \left(\omega_{\mathrm{d}}\left(\mathrm{t}-\mathrm{kT}+\frac{\mathrm{T}}{2}\right)-\theta\right)+\cos \left(\omega_{\mathrm{d}}(\mathrm{t}-\mathrm{kT})-\theta\right)\right] \\
\mathrm{kT}<\mathrm{t}<\mathrm{kT}+\frac{\mathrm{T}}{2} ; \\
-\mathrm{V}_{\mathrm{s}}+2 \mathrm{~V}_{\mathrm{s}} \frac{\omega_{0}}{\omega_{\mathrm{d}}} \mathrm{e}^{-\alpha(\mathrm{t}-\mathrm{kT})}\left[\begin{array}{l}
\mathrm{e}^{-\alpha \frac{\mathrm{T}}{2}} \cos \left(\omega_{\mathrm{d}}\left(\mathrm{t}-\mathrm{kT}+\frac{\mathrm{T}}{2}\right)-\theta\right)-\cos \left(\omega_{\mathrm{d}}(\mathrm{t}-\mathrm{kT})-\theta\right) \\
+\mathrm{e}^{\alpha \frac{\mathrm{T}}{2}} \cos \left(\omega_{\mathrm{d}}\left(\mathrm{t}-\mathrm{kT}-\frac{\mathrm{T}}{2}\right)-\theta\right) \\
, \quad \mathrm{kT}+\frac{\mathrm{T}}{2}<\mathrm{t}<(\mathrm{k}+1) \mathrm{T}
\end{array}\right]
\end{array}\right.
$$

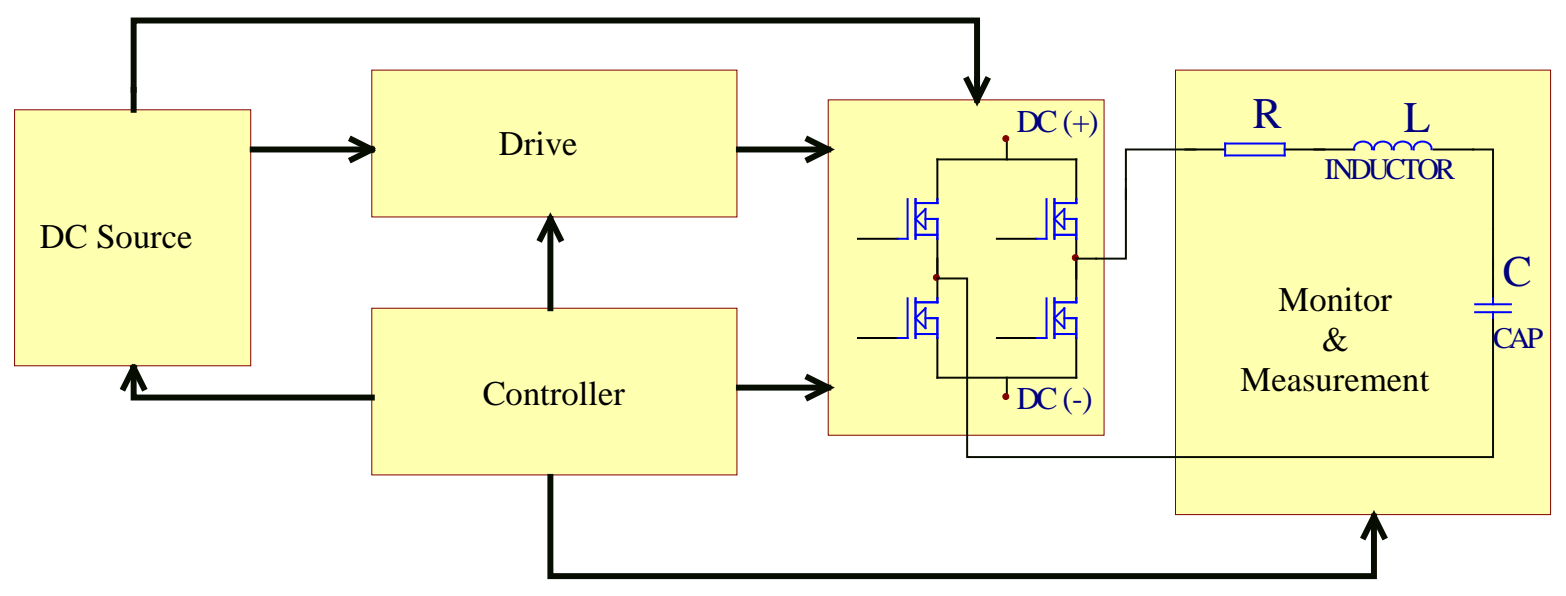


Figure 1. Circuit frame configuration of a practical experimental device.

\section{DISCUSSION}

Practical solution and device can be made as the configuration shown in Fig. 1. With a HTS, the resonant circuit can be a very high Q circuit, special aspects such as a very high voltage can be built up or a high current can be controlled. With regard to the voltage built up, practically any circuit resistance causes energy dissipation, and for each reversal of the DC source polarity for example, the corresponding increase in voltage is less than $2 V_{B}$, and the magnitude of the voltage increase gets smaller with each iteration. Any resistance in the circuit will limit the final achievable voltage. To reduce the circuit resistance to near zero leads to an infinite circuit quality factor $\mathrm{Q}$ at resonance. In a series resonant circuit with a power supply $\mathrm{V}_{\mathrm{p}}=$ $\mathrm{V}_{\mathrm{m}} \sin \omega \mathrm{t}$, the rms current $\mathrm{I}_{\max }$ in the circuit at resonance is

$$
\mathrm{I}_{\text {max }}=\mathrm{V}_{\mathrm{m}} /(\mathrm{R} \sqrt{ } 2)
$$

and the voltage across the capacitor $\mathrm{V}_{\mathrm{Cmax}}(\mathrm{rms})$ is

$$
\mathrm{V}_{\mathrm{Cmax}}=\mathrm{X}_{\mathrm{C}} \mathrm{I}_{\max }=\left(\omega_{0} \mathrm{C}\right)^{-1}\left[\mathrm{~V}_{\mathrm{m}} /(\mathrm{R} \sqrt{ } 2)\right]
$$

By assuming that the circuit resistance $\mathrm{R}$ is zero, the circuit then has infinite $Q$ at resonant frequency $\omega_{0}=1 / \sqrt{ }(\mathrm{LC}) \mathrm{rad}$ $\mathrm{s}^{-1}$. This leads to an infinite value of $\mathrm{V}_{\mathrm{C}}$ generated and $\mathrm{a}$ very large potential current. This device therefore is able to provide controls of both high voltages and high currents.

HTS technology is critical for this method to be practical. HTS wires can be used to achieve the very high Q inductor to make this method viable; on the other hand the conventional inductor technique can not make this method applicable. The high $\mathrm{Q}$ inductor is able to be realized by using HTS Ag-clad $(\mathrm{Bi}, \mathrm{Pb})_{2} \mathrm{Sr}_{2} \mathrm{Ca}_{2} \mathrm{Cu}_{3} \mathrm{O}_{10+x}$ wires, which have potential capability to make the inductor winding [3-5]. The HTS inductor virtually has no resistance for a DC current operation; however loss will be generated even at a relative low value and low frequency AC application, which however does not affect this application significantly [6].

\section{CONCLUSION}

A high $\mathrm{Q}$ resonant circuit with a power electronic controller has been verified with theoretical and also practical operation analysis, which forms a base to develop this high Q resonant method with HTS technology. The application of this method generates special aspects, such as high voltage built up and high current control, which can be readily achieved by using the newly developed HTS technology.

\section{ACKNOWLEDGMENT}

Thanks are due to A/Prof. C. Grantham, and Dr. X.P. Gu, providing help for this work. It is appreciate for the current support made by the University of Electronic Science and Technology of China, and its staff G. Zhang, H.J. Wang, H.B. Xu, S.L. Tian, L. Tong, Q. Huang, L.H. Zheng, H.L. Yan, and J.H. Zhou.

\section{REFERENCES}

[1] J.X. Jin, C. Grantham, S.X. Dou, H.K. Liu, "High voltage generation with a high $\mathrm{T}_{\mathrm{c}}$ superconducting resonant circuit", IEEE Transactions on Applied Superconductivity, Vol.7 (2), pp.881-884, 1997.

[2] J.X. Jin, S.X. Dou, F. Darmann, M. Apperley, T. Beales, "Development of an HTS inductor for an electronic high voltage generator", Physica C, Vol.341-348(1-4), pp.1601-1602, 2000.

[3] J.X. Jin, S.X Dou, H.K. Liu, C. Grantham, "Preparation of high $T_{c}$ superconducting coils for consideration of their use in a prototype fault current limiter", IEEE Transactions on Applied Superconductivity, Vol.5(2), pp.1051-1054, 1995.

[4] Y.C. Guo, J.X. Jin, H.K. Liu, Y. Tanaka, S.X. Dou, "Long lengths of silver-clad Bi2223 superconducting tapes with high current-carrying capacity”, Applied Superconductivity, Vol.5(1-6), pp.163-170, 1997.

[5] J.X. Jin, C. Grantham, H.K. Liu, S.X. Dou, “(Bi, $\mathrm{Pb})_{2} \mathrm{Sr}_{2} \mathrm{Ca}_{2} \mathrm{Cu}_{3} \mathrm{O}_{10+x} /$ Ag-clad high- $\mathrm{T}_{\mathrm{c}}$ superconducting coil and its magnetic field properties”, Philosophical Magazine B, Vol.75(6), pp.813-826, 1997.

[6] T. Hardono, C. Cook, J. X. Jin, "Measurements of ac loss in HTSC wires exposed to an alternating field using calorimetric methods", IEEE Transactions on Applied Superconductivity, Vol.9(2), pp.813816, 1999. 